\title{
Advancing Computational Reproducibility in the Dataverse Data Repository Platform
}

\author{
Ana Trisovic \\ Institute for Quantitative Social \\ Science, Harvard University \\ Cambridge, MA, USA \\ anatrisovic@g.harvard.edu \\ Gustavo Durand \\ Institute for Quantitative Social \\ Science, Harvard University \\ Cambridge, MA, USA
}

\author{
Philip Durbin \\ Institute for Quantitative Social \\ Science, Harvard University \\ Cambridge, MA, USA
}

\author{
Sonia Barbosa \\ Institute for Quantitative Social \\ Science, Harvard University \\ Cambridge, MA, USA \\ Mercè Crosas \\ Institute for Quantitative Social \\ Science, Harvard University \\ Cambridge, MA, USA \\ mcrosas@g.harvard.edu
}

\author{
Tania Schlatter \\ Institute for Quantitative Social \\ Science, Harvard University \\ Cambridge, MA, USA
}

\author{
Danny Brooke \\ Institute for Quantitative Social \\ Science, Harvard University \\ Cambridge, MA, USA
}

\begin{abstract}
Recent reproducibility case studies have raised concerns showing that much of the deposited research has not been reproducible. One of their conclusions was that the way data repositories store research data and code cannot fully facilitate reproducibility due to the absence of a runtime environment needed for the code execution. New specialized reproducibility tools provide cloud-based computational environments for code encapsulation, thus enabling research portability and reproducibility. However, they do not often enable research discoverability, standardized data citation, or long-term archival like data repositories do. This paper addresses the shortcomings of data repositories and reproducibility tools and how they could be overcome to improve the current lack of computational reproducibility in published and archived research outputs.
\end{abstract}

\section{KEYWORDS}

computational reproducibility, open data, open code, data repository, data management, data preservation.

\section{ACM Reference Format:}

Ana Trisovic, Philip Durbin, Tania Schlatter, Gustavo Durand, Sonia Barbosa, Danny Brooke, and Mercè Crosas. 2020. Advancing Computational Reproducibility in the Dataverse Data Repository Platform. In 3rd International Workshop on Practical Reproducible Evaluation of Computer Systems (P-RECS '20), June 23, 2020, Stockholm, Sweden. ACM, New York, NY, USA, 6 pages. https://doi.org/10.1145/3391800.3398173

Permission to make digital or hard copies of part or all of this work for personal or classroom use is granted without fee provided that copies are not made or distributed for profit or commercial advantage and that copies bear this notice and the full citation on the first page. Copyrights for third-party components of this work must be honored.

For all other uses, contact the owner/author(s).

P-RECS '20, June 23, 2020, Stockholm, Sweden

(C) 2020 Copyright held by the owner/author(s).

ACM ISBN 978-1-4503-7977-9/20/06.

https://doi.org/10.1145/3391800.3398173

\section{INTRODUCTION}

The requirement of reproducible computational research is becoming increasingly important and mandatory across the sciences [12]. Because reproducibility implies a certain level of openness and sharing of data and code, parts of the scientific community have developed standards around documenting and publishing these research outputs $[4,19]$. Publishing data and code as a replication package in a data repository is considered to be a best practice for enabling research reproducibility and transparency [11]. ${ }^{1}$

Some academic journals endorse this approach for publishing research outputs, and they often encourage (or require) their authors to release a replication package upon publication. Data repositories, such as Dataverse or Dryad, are the predominantly encouraged mode for sharing research data and code followed by journals' own websites (Figure 1) [6]. For example, the American Journal of Political Science (AJPS) and the journal Political Analysis have their own collections within the Harvard Dataverse repository, which is their required venue for sharing research data and code.

Recent case studies [5] reported that the research material published in data repositories does not often guarantee reproducibility. This is in part because, in their current form, data repositories do not capture all software and system dependencies necessary for code execution. Even when this information is documented by the original authors in an instructions file (like a readme), contextual information might still be missing, which could make the process of research verification and reuse hard or impossible. This is also often the case with some of the alternative ways of publishing research data and code, for example, through the journal's website. A study [17] reported that the majority of supplemental data deposited on a journal's website was inaccessible due to broken links. Such problems are less likely to happen in data repositories

\footnotetext{
${ }^{1}$ At Dataverse, the data and code used to reproduce a published study are called "replication data" or a "replication package"; in Whole Tale, this is called a "tale", and in Code Ocean a "reproducible capsule".
} 


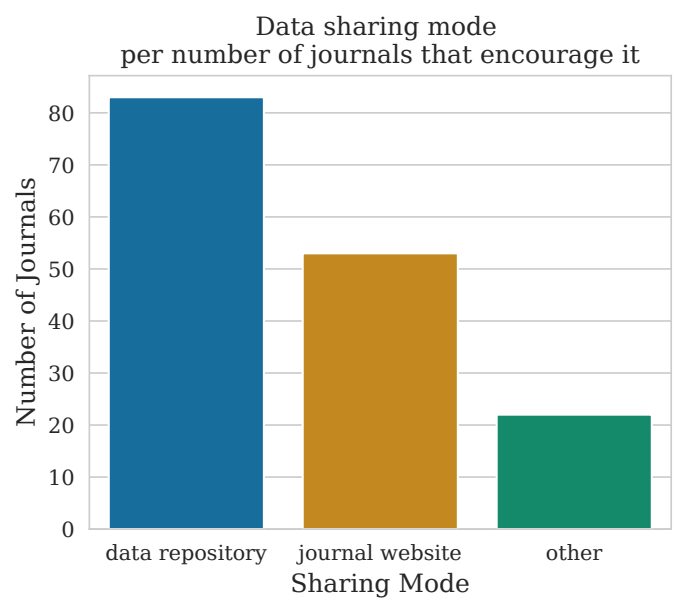

Figure 1: Aggregated results for the most popular data sharing mode in economics, political science, psychology, anthropology, sociology, and history from Ref. [6].

that follow standards for long-term archival and support persistent identifiers.

Some researchers prefer to release their data and code on personal websites or websites like GitHub and GitLab. This approach does not natively provide a standardized persistent citation for referencing and accessing the research materials, nor sufficient metadata to make it discoverable in data search engines like Google Dataset Search and DataCite search. In addition, it does not guarantee long-term accessibility as data repositories do. Because research deposited this way does not typically contain runtime environment, system or contextual information, this approach is also often ineffective in enabling computational reproducibility [16].

New cloud-based platforms have emerged to support research data organization, collaborative work, and reproducibility [14]. Even though the number of different and useful reproducibility tools is constantly increasing, in this paper, we are going to focus on the following projects: Code Ocean [18], Whole Tale [1], Renku ${ }^{2}$ and Binder $[8,9]$. All of these tools are available through a web browser, and they are based on the containerization technology Docker, which provides a standardized way to capture the computational environment that can be shared, reproduced, and reused.

(1) Code Ocean is a research collaboration platform that enables its users to develop, execute, share, and publish their data and code. The platform supports a large number of programming languages including $\mathrm{R}, \mathrm{C} / \mathrm{C}++$, Java, Python, and it is currently the only platform that supports code sharing in proprietary software like MATLAB and Stata.

(2) Whole Tale is a free and open-source reproducibility platform that, by capturing data, code, and a complete software environment, enables researchers to examine, transform and republish research data that was used in an academic article.

\footnotetext{
${ }^{2}$ https://renkulab.io
}

(3) Renku is a project similar to Whole Tale that focuses on employing tools for best coding practices to facilitate collaborative work and reproducibility.

(4) Binder is a free and open-source project that allows users to run notebooks (Jupyter or R) and other code files by creating a containerized environment using configuration files within a replication package (or a repository).

Even though virtual containers are currently considered the most comprehensive way to preserve computational research $[7,15]$, they do not entirely comply with modern scientific workflows and needs. Through the use of containers, the reproducibility platforms in most cases fail to support FAIR principles (Findable, Accessible, Interoperable, and Reusable) [19], standardized persistent citation, and long-term preservation of research outputs as data repositories strive to do. Findability is enabled through standard or communityused metadata schemas that document research artifacts. Data and code stored in a Docker container on a reproducibility platform are not easily visible nor accessible from outside of the container, which thus hinders their findability. This could be an issue for a researcher looking for a specific dataset rather than a replication package. In addition, unlike data repositories, reproducibility platforms do not undertake a commitment to the archival of research materials. This means that, for example, in a scenario where a reproducibility platform runs out of funding, the deposited research could become inaccessible.

Individually neither data repositories nor reproducibility platforms cannot fully support scientific workflows and requirements for reproducibility and preservation. This paper explains how their shortcomings could be overcome through integration that would result in a robust paradigm for preserving computational research and enabling reproducibility and reuse while making the replication packages FAIR. We argue that through the integration of these existing projects, rather than the invention of new ones, we could effectively combine their complementary functionalities.

\section{RELATED WORK}

Through integration, reproducibility platforms and data repositories create a synergy that addresses weaknesses of both approaches. Some of these integrations are already on the way:

- CLOCKSS ${ }^{3}$ is an archiving repository that preserves data with regular validity checks. Unlike other data repositories, it does not provide public or user access to the preserved content, except in special cases that are referred to as "triggered content". Code Ocean has partnered with CLOCKSS to preserve in perpetuity research capsules associated with publications from some of the collaborating journals.

- The Whole Tale platform relies on integrations with external resources for long-term stewardship and preservation. They already enable data import from data repositories, and a publishing functionality for a replication package is currently underway through DataONE, Dataverse, and Zenodo [3].

- Stencila is an open-source office suite designed for creating interactive, data-driven publications. With its familiar user interface, it is geared toward users of Microsoft Word and Excel. It integrates data and code as self-contained parts of

\footnotetext{
${ }^{3}$ https://clockss.org
} 


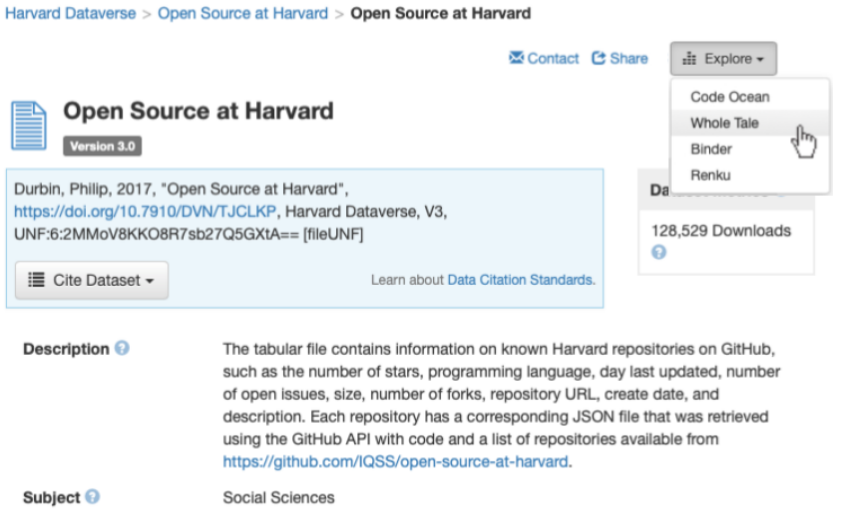

Figure 2: Preliminary view of the "Explore" button on the dataset landing page in Dataverse that enables exploring replication packages in the reproducibility platforms.

the publication, while also enabling external researches to explore the data and write custom code. Stencila and the journal eLife have partnered up to facilitate reproducible publications [10].

\section{IMPLEMENTATION}

In this paper, we present our developments in the context of the Dataverse Project, which is a free and open-source software platform to archive, share, and cite research data. Currently, 59 institutions around the globe run Dataverse instances as their data repository.

Dataverse's integration with the reproducibility platforms has propelled a series of questions and developments around advancing reproducibility for its vast and diverse user community. First, while container files can be uploaded to Dataverse, there is no special handling for these files, which can result in mixed outcomes for researchers trying to verify reproducibility. Second, it is important to facilitate the capture of computational dependencies for Dataverse users who choose not to use a reproducibility platform. Finally, for a replication package with multiple seemingly disorganized code files, it is essential to minimize the time and effort of an external user who wants to rerun and reuse the files. Therefore, new functionality to support container-based deposits, organization, and access needs to be added to Dataverse to improve reproducibility.

\subsection{Integration with reproducibility platforms}

Dataverse integration with reproducibility platforms should allow both adding new research material into Dataverse, and importing and reusing the existing material from Dataverse into a reproducibility platform. This communication would be implemented through a series of existing and new APIs. The reproducibility platforms that have ongoing integration collaborations with Dataverse are Code Ocean, Whole Tale, Binder, and Renku.

Importing research material from Dataverse means that data and code that already exist in Dataverse could be transferred directly into a reproducibility platform. On the Dataverse side, this is implemented through a new button "Explore", shown in Figure 2. When

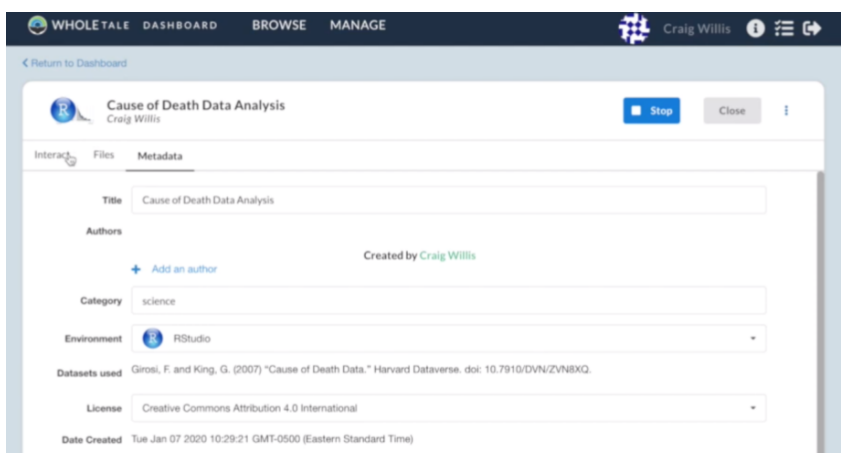

Figure 3: Using data from Dataverse in the Whole Tale environment. Snapshot from YouTube video https://www.youtube.com/watch?v=oWEcFpEUmrU. Credit: Craig Willis.

the button is clicked, the replication package is copied and sent to a reproducibility platform where it, using the configuration files from the package, creates a Docker container, places all data and code into it and provides a view through a web browser. This means that Dataverse users will not need to download any of the files to their personal computers or set up a computational environment to execute and explore the deposited files. So far, the "Explore" button is functional for the Whole Tale platform, while the others are underway. ${ }^{4}$

The researcher whose starting point is a reproducibility platform will be able to import materials for their analysis directly from Dataverse. An example where a researcher is importing Dataverse open data as "external data" into Whole Tale is shown in Figure 3, as this integration is now implemented. Similarly, Figure 4 shows new integration developments with the lightweight cloud platform, Binder, that now enables the users to import and view data from Dataverse. The export of the encapsulated research material into Dataverse will also be possible, which means that, once an analysis is ready for dissemination, the researchers would initiate "analysis export" in a reproducibility platform, that would then copy the files from a Docker container in a single compressed file and send them to Dataverse. This way, all necessary computational dependencies are automatically recorded by the reproducibility platform and stored at a data repository following preservation standards. This functionality is already implemented in Renku. ${ }^{5}$

\subsection{Handling containers}

Importing replication packages from reproducibility platforms means that Dataverse would need to support the capture of their virtual containers. Since all aforementioned reproducibility platforms are based on the Docker containerization technology, new Dataverse developments focus on Docker containers. Docker containers can be built automatically from the instructions laid out in a "Dockerfile", which is an often tiny text file that contains commands, typically

\footnotetext{
${ }^{4}$ Dataverse documentation for integrations: http://guides.dataverse.org/en/4.20/admin/ external-tools.html

${ }^{5}$ Integration code at https://github.com/SwissDataScienceCenter/renkupython/pull/909
} 


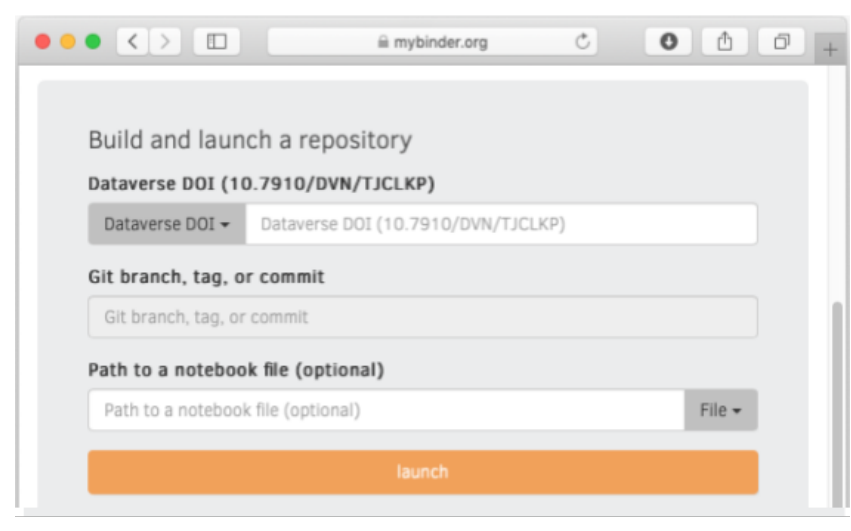

Figure 4: Binder's GUI (https://mybinder.org) supports viewing content from Dataverse.

for installing software and dependencies, to set up a runtime environment needed for research analysis. The Dataverse platform will encourage depositing Dockerfiles to capture the computational environment. This would allow users to explore replication packages in any supported reproducibility platform as Dockerfiles are agnostic to their base infrastructures. An alternative solution would be to create a Dataverse Docker registry where the whole images would be preserved. This approach will not be pursued at the time due to excessive storage requirements.

It is important to mention that at present, any file can be stored at Dataverse, including a Dockerfile, and that there are currently dozens of Dockerfiles stored at Harvard's instance of Dataverse. However, whereas previously Dockerfiles were considered as "other files", in the new development they will be pre-identified at upload, and thus will require additional metadata. When a reproducibility platform automatically generates a Dockerfile, it is likely to be suitable for portability and preservation. However, when a researcher prepares it, this might not be the case. Dockerfiles could be susceptible to some of the practices that cause irreproducibility, like the absence of software dependency versions or the use of absolute (fixed) file paths, which is why Dataverse will encourage its users to use best practices when preparing and depositing these files. ${ }^{6}$

\subsection{Capturing execution commands}

In addition to capturing a Docker container via Dockerfile, it is important to capture the sequence of steps that the user ran to obtain their results. This applies to the results obtained with commandline languages such as Python, MATLAB, Julia. Capturing the command sequence is particularly important when there are multiple code files within the replication dataset without the clear notation in which order they should be executed. The commands will be captured in the replication package metadata using a community standard to be determined (see, for example, RO-Crate [2]).

In case a replication package was imported into Dataverse from a reproducibility platform, its replication commands would be automatically populated. For example, Code Ocean generates the

\footnotetext{
${ }^{6}$ There are other known limitations of Docker when it comes to reproducibility, like the lack of determinism [13] and inability to describe computations done in HPC, HTC or cloud environments, however, that discussion is beyond the scope of this paper.
}

commands that build and run a Docker container for each replication package, and it also encourages researchers to specify the command sequence in a file called "run" to automatize their code. This means that all command sequences that run "outside" and "inside" the container are captured. Dataverse users who choose not to use a reproducibility platform would need to manually specify this sequence based on presented best practices.

\subsection{Improving FAIR-ness}

Because no dataset would be "hidden" within a virtual container at Dataverse, all files originally used in research would be indexed and thus findable by one of the common dataset search engines. They would also be accessible directly from the dataset landing page on the web. Their interoperability and reusability would be now improved with the integration with the reproducibility platforms, as the barriers to creating a runtime environment and running code files would be alleviated.

\subsection{New data metrics}

Dataverse traditionally aims to provide incentives to researchers to share data through data citation credit, data metrics (e.g. a count of downloads for datasets) and access requests for restricted data. One of the completed new developments includes integrating certifications or science badges, such as Open Data and Open Materials, within a dataset landing page on Dataverse.

The new support for reproducibility tools and containers will also result in creating new metrics for the users. The datasets that are deposited through a reproducibility platform into Dataverse will be denoted with a "reproducibility certification" badge that will signal their origin and easy execution on the cloud. For example, a replication package that was received from a reproducibility platform Whole Tale, will include its origin information and encourage its exploration and reuse through Whole Tale.

\section{FUNCTIONALITY AND USE-CASES}

Dataverse integration with reproducibility platforms and new developments that improve the reproducibility of deposited research will facilitate research workflows relating to verification, preservation, and reuse in the following ways (shown in Figure 5):

(1) Encapsulate research: The first supported workflow enables authors to deposit their data and code through Code Ocean, Whole Tale, or Renku, which then create a replication package that is sent for dissemination and preservation to Dataverse. Dataverse users who were not previously familiar with Docker containerization technology will now be able to containerize their research through the new workflow. In addition, this workflow is particularly important for prestigious academic journals that verify research reproducibility through third-party curation services and a reproducibility platform. For example, code review at the journal Political Analysis, which collaborates with Code Ocean and Harvard Dataverse for data dissemination and preservation, will be significantly sped up with the deployment of this workflow, as all the code associated with a publication will already be automatized, containerized and available on the cloud. 


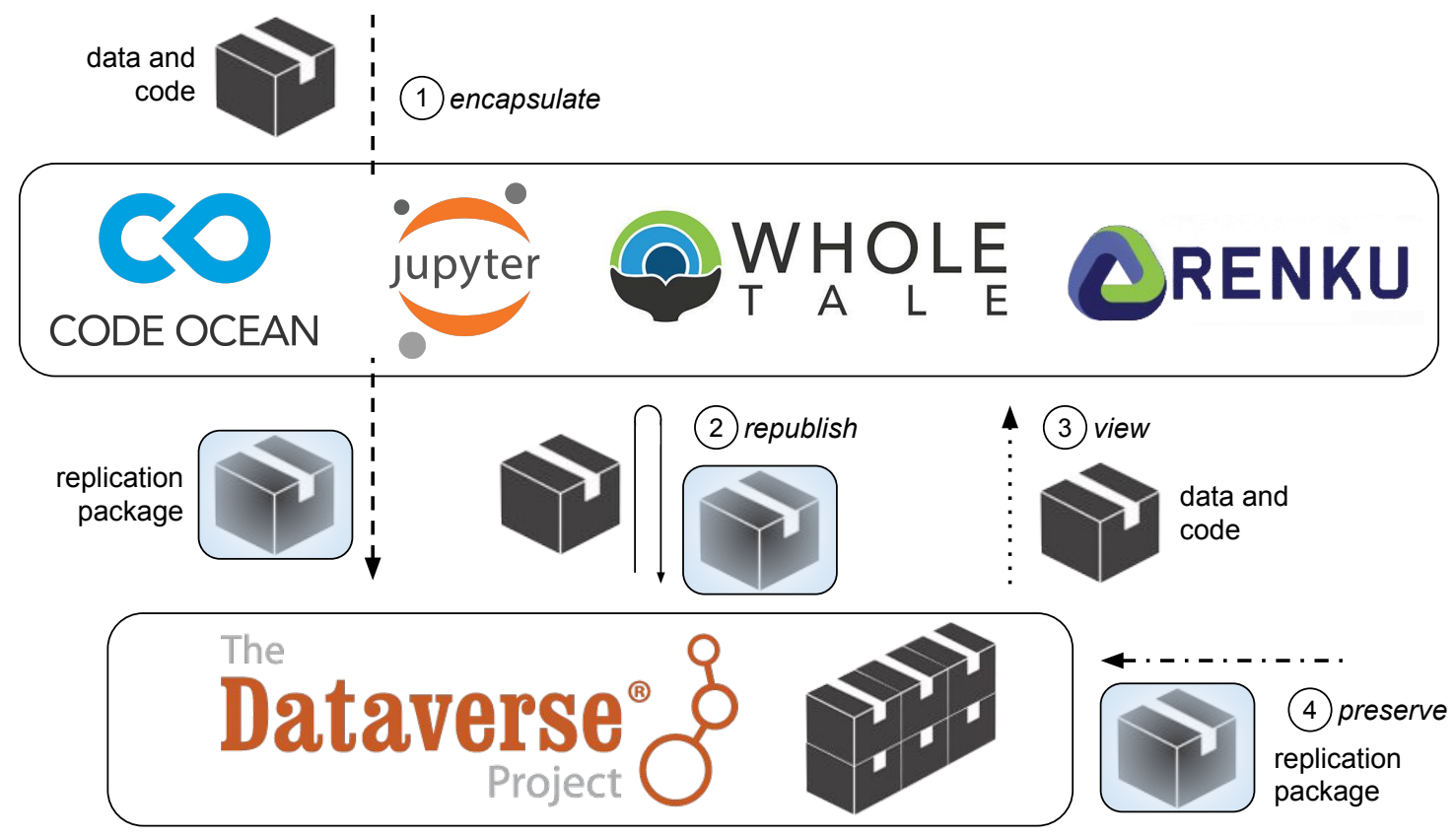

Figure 5: Four main workflows that Dataverse aims to support with reproducibility platform integration.

(2) Modify and republish research: The second workflow covers pulling a replication package from Dataverse and republishing it after an update. This would create a new version of the package in Dataverse, as well as track provenance about the original package. Peer-review and revisions of the package should thus be much easier. In addition, the replication packages on Dataverse that currently do not have information on their runtime environment could be updated and republished with a Dockerfile generated by one of the reproducibility platforms.

(3) View deposited research materials: The third functionality allows viewing and exploring the content of deposited research without the need to download the files and install new software. This could be particularly valuable for external researchers and students who would like to understand research results or reuse data or code.

(4) Preserve computational environment with Dockerfile: Through new developments in Dataverse that encourage depositing Dockerfiles with best practices, the researchers who are experienced in using Docker will now be able to adequately preserve these files in the repository.

\section{CONCLUSIONS}

In the last decade, there has been extensive discussion around preservation, reproducibility, and openness of computational research, which has resulted in creating multiple new tools to facilitate these efforts, the most popular being data repositories and reproducibility platforms. Individually these two efforts cannot fully facilitate findable, interoperable, reusable, and reproducible research materials, however, this paper presents a robust solution achievable through their integration.
Described integrations have resulted in developing new functionality in Dataverse, such as expanding on the existing API, introducing new replication-package metadata, and handling virtual containers via Dockerfile. In addition to allowing research preservation in a reproducible and reusable way through the integrations, Dataverse aims to identify new and useful data metrics to be displayed on the dataset landing page. Due to the fact that there is an increasing number of similar reproducibility tools, this paper also advocates for considering integration with an existing solution before (re)inventing a new reproducibility tool.

\section{ACKNOWLEDGMENTS}

Thank you to the Dataverse team for their help and support. This work is partially funded by the Sloan Foundation (award number G-2018-11111). Ana Trisovic is funded by the Sloan Foundation.

\section{REFERENCES}

[1] Adam Brinckman et al. 2019. Computing environments for reproducibility: Capturing the Whole Tale. Future Generation Computer Systems 94 (2019), 854867.

[2] Eoghan Ó Carragáin, Carole Goble, Peter Sefton, and Stian Soiland-Reyes. [n.d.]. RO-Crate, a lightweight approach to Research Object data packaging. ([n. d.]).

[3] Kyle Chard, Niall Gaffney, Matthew B Jones, Kacper Kowalik, Bertram Ludäscher, Jarek Nabrzyski, Victoria Stodden, Ian Taylor, Matthew J Turk, and Craig Willis. 2019. Implementing computational reproducibility in the Whole Tale environment. In Proceedings of the 2nd International Workshop on Practical Reproducible Evaluation of Computer Systems. 17-22.

[4] Xiaoli Chen et al. 2019. Open is not enough. Nature Physics 15, 2 (2019), 113-119.

[5] Christian Collberg and Todd A Proebsting. 2016. Repeatability in computer systems research. Commun. ACM 59, 3 (2016), 62-69.

[6] Mercè Crosas, Julian Gautier, Sebastian Karcher, Dessi Kirilova, Gerard Otalora, and Abigail Schwartz. 2018. Data policies of highly-ranked social science journals. (2018).

[7] Ivo Jimenez, Carlos Maltzahn, Adam Moody, Kathryn Mohror, Jay Lofstead, Remzi Arpaci-Dusseau, and Andrea Arpaci-Dusseau. 2015. The role of container 
technology in reproducible computer systems research. In 2015 IEEE International Conference on Cloud Engineering. IEEE, 379-385.

[8] P Jupyter, M Bussonnier, J Forde, J Freeman, B Granger, T Head, C Holdgraf, K Kelley, G Nalvarte, A Osheroff, et al. 2018. Binder 2.0-Reproducible, interactive, sharable environments for science at scale. In Proceedings of the 17th python in science conference, Vol. 113. 120.

[9] Thomas Kluyver, Benjamin Ragan-Kelley, Fernando Pérez, Brian E Granger Matthias Bussonnier, Jonathan Frederic, Kyle Kelley, Jessica B Hamrick, Jason Grout, Sylvain Corlay, et al. 2016. Jupyter Notebooks-a publishing format for reproducible computational workflows.. In ELPUB. 87-90.

[10] Giuliano Maciocci, Michael Aufreiter, and Nokome Bentley. 2019. Introducing eLife's first computationally reproducible article. eLife Labs [Internet] 20 (2019).

[11] Jennifer C Molloy. 2011. The open knowledge foundation: open data means better science. PLoS biology 9, 12 (2011), e1001195.

[12] Engineering National Academies of Sciences, Medicine, et al. 2019. Reproducibility and replicability in science. National Academies Press.

[13] Omar S Navarro Leija, Kelly Shiptoski, Ryan G Scott, Baojun Wang, Nicholas Renner, Ryan R Newton, and Joseph Devietti. 2020. Reproducible Containers. In
Proceedings of the Twenty-Fifth International Conference on Architectural Support for Programming Languages and Operating Systems. 167-182.

[14] JM Perkel. 2019. Make code accessible with these cloud services.

[15] Stephen R Piccolo and Michael B Frampton. 2016. Tools and techniques for computational reproducibility. GigaScience 5, 1 (2016), 30.

[16] João Felipe Pimentel, Leonardo Murta, Vanessa Braganholo, and Juliana Freire. 2019. A large-scale study about quality and reproducibility of jupyter notebooks. In Proceedings of the 16th International Conference on Mining Software Repositories. IEEE Press, 507-517.

[17] Anisa Rowhani-Farid and Adrian G Barnett. 2018. Badges for sharing data and code at Biostatistics: an observational study. F1000Research 7 (2018).

[18] Thomas Staubitz, Hauke Klement, Ralf Teusner, Jan Renz, and Christoph Meinel. 2016. CodeOcean-A versatile platform for practical programming excercises in online environments. In 2016 IEEE Global Engineering Education Conference (EDUCON). IEEE, 314-323.

[19] Mark D Wilkinson et al. 2016. The FAIR Guiding Principles for scientific data management and stewardship. Scientific data 3 (2016). 